To appear in Local Structure from Diffraction, Fundamental Materials Science Series, eds. M.F. Thorpe and S.J.L. Billinge, Plenum Press, New York, 1998.

\title{
NON-MEAN-FIELD THEORIES OF SHORT RANGE ORDER AND DIFFUSE SCATTERING ANOMALIES IN DISORDERED ALLOYS
}

\author{
Igor Tsatskis用 \\ Department of Earth Sciences \\ University of Cambridge \\ Downing Street \\ Cambridge CB2 3EQ \\ United Kingdom
}

\section{INTRODUCTION}

Local, or short-range, order in disordered alloys is an important and exciting phenomenon which is quantified in electron, X-ray and neutron scattering experiments. It is discussed in many excellent reviews and books, ${ }^{1-6}$ as well as in the multitude of original research papers.

This relatively short review of the subject does not attempt to discuss all aspects of the problem of local correlations in alloys. In particular, we will not touch such issues as multiatom (cluster) interactions, static displacements and vibrations of alloy atoms, partially ordered, multicomponent or amorphous alloys. As a result, we will concentrate on the Hamiltonian traditional for the considered problem, that of the Ising model on a rigid ideal lattice with pair, but otherwise arbitrary (i.e., of any range) interatomic interactions.

The central object of the paper is the pair correlation function of the corresponding dynamical variables of the model, the occupation numbers or spin variables, the Fourier transform of which is proportional to the intensity of diffuse scattering caused by atomic short-range order. The main aim is to show that the expression for this quantity has certain internal structure analogous, e.g., to that of the averaged Green's function used in the electronic theory of disordered alloys. This structure is independent of the approximation used for the quantitative description of correlations. As will be seen, this structure alone, without further specification of a particular theory of short-range order, allows us to see new possibilities in diffuse scattering, some of which have recently

\footnotetext{
${ }^{*}$ Former name: I.V. Masanskii
} 
been observed experimentally.

The present contribution is organized as follows. First two sections are auxiliary and serve mainly for reference purposes; the former introduces relation between the phenomenological Hamiltonian of binary solid solutions and the Ising model, as well as necessary definitions and formulae, while the latter describes briefly standard approaches to the theory of electronic structure of disordered alloys. Readers familiar with the material contained in these sections can skip it and proceed directly to the third section where the key expression (75) for the diffuse intensity is introduced. In the fourth section its derivation and relation with alloy thermodynamics are discussed. Without any doubt, many readers would quickly realize that Eq. (75) is simply one of the possible forms of the famous Dyson equation. Such readers can then focus on the two last sections. The fifth section reviews, from the point of view adopted in this paper, existing theoretical approaches - both traditional and relatively new - to the problem of calculation of the pair correlation function and the diffuse-scattering intensity. Finally, in the sixth section based on the recent author's work it is shown how the internal structure of Eq. (75) leads to understanding existing and predicting new diffuse-scattering anomalies.

\section{LIST OF ABBREVIATIONS}

$\begin{array}{ll}\text { AE } & \text { alpha-expansion } \\ \text { ATA } & \text { average } t \text {-matrix approximation } \\ \text { CPA } & \text { coherent potential approximation } \\ \text { GEM } & \text { gamma-expansion method } \\ \text { HTE } & \text { high-temperature expansion } \\ \text { KCM } & \text { Krivoglaz-Clapp-Moss (approximation) } \\ \text { MC } & \text { Monte Carlo (simulation) } \\ \text { MFA } & \text { mean-field approximation } \\ \text { PCF } & \text { pair correlation function } \\ \text { RPA } & \text { random-phase approximation } \\ \text { SM } & \text { spherical model } \\ \text { SRO } & \text { short-range order } \\ \text { SSA } & \text { single-site approximation } \\ \text { VCA } & \text { virtual crystal approximation }\end{array}$

\section{DESCRIPTION OF A BINARY ALLOY}

We will consider the standard model of a binary alloy used in the statistical theory of ordering. ${ }^{1-6}$ In this model two sorts of atoms (A and B) are distributed over $N$ sites of a rigid lattice; there are no vacancies or other lattice defects. For simplicity reasons the consideration is confined to the case of the lattice with one site per unit cell. All sites of the lattice are equivalent, i.e., there are no distinct sublattices; this situation corresponds to disorder or ferromagnetic ordering. A particular alloy configuration is fully described by the set of occupation numbers $p_{i}^{\alpha}$,

$$
p_{i}^{\alpha}= \begin{cases}1, & \text { atom of type } \alpha \text { at the lattice site } i \\ 0, & \text { otherwise }\end{cases}
$$


The occupation numbers satisfy obvious relations

$$
\begin{aligned}
\sum_{\alpha} p_{i}^{\alpha} & =1, \\
\sum_{i} p_{i}^{\alpha} & =N^{\alpha}, \\
\sum_{\alpha} N^{\alpha} & =N,
\end{aligned}
$$

where $N^{\alpha}$ is the total number of atoms of type $\alpha$, and the last equation holds because the total number of alloy atoms is equal to the total number of lattice sites. Indices $\alpha$ and $\beta$ take only two values, $\mathrm{A}$ or $\mathrm{B}$. It is assumed that the interatomic interactions are pairwise; an atom of type $\alpha$ at site $i$ interacts with an atom of type $\beta$ at site $j$ with a potential $V_{i j}^{\alpha \beta}$. Then the configurational part of the alloy Hamiltonian is

$$
H=\frac{1}{2} \sum_{i j} \sum_{\alpha \beta} V_{i j}^{\alpha \beta} p_{i}^{\alpha} p_{j}^{\beta} .
$$

The main aim of statistical mechanics is the calculation of the partition function ${ }^{7}$

$$
Z=\operatorname{Tr} \exp (-\beta H),
$$

where $\beta=1 / k_{B} T, k_{B}$ is the Boltzmann constant, $T$ the absolute temperature, and Tr denotes the trace of a matrix. It is convenient to avoid restriction (3) on the total number of atoms of each type and work in the grand-canonical ensemble, calculating the grand partition function $\tilde{Z}$,

$$
\tilde{Z}=Z \exp \left(\beta \sum_{\alpha} \mu^{\alpha} N^{\alpha}\right),
$$

where $\mu^{\alpha}$ is the chemical potential of atoms $\alpha$. In this approach the number of atoms is a function of the corresponding chemical potential, and after having done the calculation the chemical potential is adjusted to get the required number of atoms. To unify canonical and grand-canonical treatments, i.e., to get the same formula (6) for $\tilde{Z}$, an effective Hamiltonian $\tilde{H}$ is often introduced:

$$
\tilde{H}=H-\sum_{\alpha} \mu^{\alpha} N^{\alpha} .
$$

The expression for the effective Hamiltonian is then

$$
\tilde{H}=\frac{1}{2} \sum_{i j} \sum_{\alpha \beta} V_{i j}^{\alpha \beta} p_{i}^{\alpha} p_{j}^{\beta}-\sum_{i} \sum_{\alpha} \mu^{\alpha} p_{i}^{\alpha} .
$$

As is well-known, the statistical-mechanical problem defined by the Hamiltonian (9) can be transformed into the equivalent problem of an Ising magnet in a magnetic field. The occupation numbers are not yet all independent, due to Eq. (2). Dependent variables can be eliminated by introducing a spin variable $s_{i}$,

$$
s_{i}= \begin{cases}+1, & \text { spin up }(\operatorname{atom} A) \text { at the site } i \\ -1, & \text { spin down }(\operatorname{atom} B) \text { at the site } i .\end{cases}
$$

The relations between the occupation numbers and the spin variables are

$$
\begin{aligned}
p_{i}^{A} & =\frac{1}{2}\left(1+s_{i}\right), \\
p_{i}^{B} & =\frac{1}{2}\left(1-s_{i}\right) .
\end{aligned}
$$


Inserting Eqs. (11) and (12) into the expression (9) for the effective Hamiltonian $\tilde{H}$, we find that, apart from the configuration-independent term, the Hamiltonian is that of the Ising model,

$$
\tilde{H}=-\frac{1}{2} \sum_{i j} J_{i j} s_{i} s_{j}-h \sum_{i} s_{i},
$$

where the effective exchange integral $J_{i j}$ and the magnetic field $h$ are given by

$$
\begin{aligned}
J_{i j} & =-\frac{1}{2} V_{i j}, \\
h & =\frac{1}{2}\left(\mu^{A}-\mu^{B}\right)-\frac{1}{4} \sum_{j}\left(V_{i j}^{A A}-V_{i j}^{B B}\right), \\
V_{i j} & =\frac{1}{2}\left(V_{i j}^{A A}+V_{i j}^{B B}\right)-V_{i j}^{A B},
\end{aligned}
$$

and $V_{i j}$ is the pair ordering potential. The second term in Eq. (15) does not, in fact, depend on the index $i$ because all interatomic potentials are functions of only the distance between interacting atoms.

For the following we define concentrations $c^{\alpha}$ and magnetization $m$ which are statistical averages of the occupation numbers (1) and the spin variables (10), respectively,

$$
\begin{aligned}
c^{\alpha} & =\left\langle p_{i}^{\alpha}\right\rangle, \\
m & =\left\langle s_{i}\right\rangle,
\end{aligned}
$$

getting from Eqs. (11) and (12)

$$
c=\frac{1}{2}(1+m)
$$

where $c=c^{A}=1-c^{B}$. Here the symbol $\langle\ldots\rangle$ denotes the statistical averaging with the effective Hamiltonian $\tilde{H}$, Eq. (9) or (13),

$$
\langle\ldots\rangle=\frac{\operatorname{Tr}[\ldots \exp (-\beta \tilde{H})]}{\operatorname{Tr} \exp (-\beta \tilde{H})}
$$

We also introduce irreducible PCFs for the occupation numbers,

$$
G_{i j}^{\alpha \beta}=\left\langle p_{i}^{\alpha} p_{j}^{\beta}\right\rangle-\left\langle p_{i}^{\alpha}\right\rangle\left\langle p_{j}^{\beta}\right\rangle
$$

and for the spin variables,

$$
G_{i j}=\left\langle s_{i} s_{j}\right\rangle-\left\langle s_{i}\right\rangle\left\langle s_{j}\right\rangle \text {. }
$$

The notion of irreducibility comes from the diagram technique for the Ising model, in the framework of which it can be shown that the irreducible PCF does not contain so-called split diagrams which are present in the expansion for the correlation function $\left\langle s_{i} s_{j}\right\rangle{ }^{8}$ Using relations (11) and (12) between the occupation numbers and the spin variables, it is easy to obtain the correspondence between the PCFs (21) and (22),

$$
G_{i j}^{A A}=G_{i j}^{B B}=-G_{i j}^{A B}=\frac{1}{4} G_{i j}
$$

and, therefore, there exists only one independent PCF for the occupation numbers. In the theory of alloys the Warren-Cowley parameters $\alpha_{i j}$, instead of the PCFs (21), are traditionally used for the description of SRO. They are defined as

$$
\alpha_{i j}=1-\frac{\left\langle p_{i}^{A} p_{j}^{B}\right\rangle}{c(1-c)},
$$


and, according to Eqs. (17), (19), (21) and (23),

$$
G_{i j}=4 c(1-c) \alpha_{i j}=\left(1-m^{2}\right) \alpha_{i j} .
$$

The diagonal matrix elements of the PCFs (21) and (22) can be expressed in terms of the averages (17) and (18). Taking into account identities $\left(p_{i}^{\alpha}\right)^{2}=p_{i}^{\alpha}$ and $s_{i}^{2}=1$ which follow from the definitions (1) and (10), we have

$$
\begin{aligned}
G_{i i}^{A A}=G_{i i}^{B B} & =-G_{i i}^{A B}=c(1-c) \\
G_{i i} & =1-m^{2} \\
\alpha_{i i} & =1
\end{aligned}
$$

The last equation leads to the important sum rule in the reciprocal space. Let us introduce the lattice Fourier transformation,

$$
\begin{aligned}
f(\mathbf{k}) & =\frac{1}{N} \sum_{i j} f_{i j} \exp \left(i \mathbf{k} \mathbf{r}_{i j}\right) \\
f_{i j} & =\frac{1}{\Omega} \int d \mathbf{k} f(\mathbf{k}) \exp \left(-i \mathbf{k} \mathbf{r}_{i j}\right),
\end{aligned}
$$

where $f_{i j}$ is an arbitrary lattice function, $\mathbf{k}$ the wavevector, $\mathbf{r}_{i j}=\mathbf{r}_{i}-\mathbf{r}_{j}, \mathbf{r}_{i}$ the radiusvector of the site $i$, and the integration in Eq. (30) is carried out over the Brillouin zone of volume $\Omega$. Eq. (28) can then be rewritten as

$$
\frac{1}{\Omega} \int d \mathbf{k} \alpha(\mathbf{k})=1
$$

Recalling that the Fourier transform $\alpha(\mathbf{k})$ of the SRO parameters $\alpha_{i j}$ is the SRO part of the diffuse-scattering intensity measured in Laue units, ${ }^{5}$ we conclude that the sum rule (31) expresses the property of conservation of the integrated SRO intensity.

\section{ELECTRONIC THEORY OF DISORDERED ALLOYS}

In what follows we will occasionally turn to ideas which were developed in the theory of electronic structure of disordered alloys. ${ }^{2,6,9-11}$ With this in mind, we deviate now from the main theme of this paper and consider briefly (and rather formally, without discussing the physical meaning of derived formulae) methods of calculating the Green's function of electrons averaged over possible alloy configurations. There is some overlapping of notations used in this section with those in the rest of the paper; however, the present section is quite isolated from the statistical-mechanical part of the discussion, and hence this overlapping should not lead to any confusion.

The central role in the one-electron theory of disordered alloys is played by the electronic Green's function $G$ which may be defined as a resolvent of the alloy Hamiltonian $H$

$$
G=(E-H)^{-1},
$$

where $E$ is the energy of an electron. The Hamiltonian of a disordered binary alloy is usually assumed to be a sum of two operators,

$$
H=H_{0}+V \text {. }
$$

The first of these two terms, $H_{0}$, is translationally invariant, while the second, the random one-electron potential $V$, depends on particular alloy configuration and is usually 
assumed to be diagonal in the site representation. The operator $V$ is a sum of individual potentials $V_{i}$ centred at each site $i$ and acquiring two possible values, $V^{A}$ and $V^{B}$, in accordance with the type of atom occupying the considered site.

\section{Propagator expansion}

The potential $V$ is often viewed as a perturbation, though not necessarily small, of the initial unperturbed Hamiltonian $H_{0}$. Defining the unperturbed Green's function,

$$
G_{0}=\left(E-H_{0}\right)^{-1}
$$

one can easily construct the Dyson equation,

$$
G=G_{0}+G_{0} V G
$$

Iterating this equation and averaging over all possible alloy configurations (the averages are denoted by brackets), we get the propagator expansion

$$
\langle G\rangle=G_{0}+G_{0}\langle V\rangle G_{0}+G_{0}\left\langle V G_{0} V\right\rangle G_{0}+\ldots
$$

The averaged Green's function satisfies another Dyson equation,

$$
\langle G\rangle=G_{0}+G_{0} \Sigma\langle G\rangle
$$

where the operator $\Sigma$ is called the self-energy. The self-energy is, in terms of the diagrammatic expansion for the averaged Green's function $\langle G\rangle$ generated by Eq. (36), the irreducible part of $\langle G\rangle$, i.e., the sum of all graphs for $\langle G\rangle$ which cannot be separated into two parts by cutting a single bare-propagator line $G_{0}$. From Eq. (37) it follows that

$$
\langle G\rangle=\left(G_{0}^{-1}-\Sigma\right)^{-1}=\left(E-H_{0}-\Sigma\right)^{-1},
$$

the second equation comes from Eq. (34).

\section{Locator expansion}

There exists another perturbation series, the locator expansion, which is the expansion in powers of the unperturbed Hamiltonian $H_{0}$ rather than the potential $V$. In this case the unperturbed Green's function is that of the localized atomic states,

$$
g=(E-V)^{-1}
$$

and the corresponding Dyson equation has the form

$$
G=g+g H_{0} G
$$

As in the case of the propagator expansion, we iterate this equation and average over

the ensemble of configurations term by term, obtaining the following expansion:

$$
\langle G\rangle=\langle g\rangle+\left\langle g H_{0} g\right\rangle+\left\langle g H_{0} g H_{0} g\right\rangle+\ldots .
$$

By introducing the so-called fully renormalized interactor $U$,

$$
U=H_{0}+H_{0} G H_{0},
$$


full formal analogy between the propagator (Eq. (36)) and locator expansions is achieved:

$$
\langle U\rangle=H_{0}+H_{0}\langle g\rangle H_{0}+H_{0}\left\langle g H_{0} g\right\rangle H_{0}+\ldots .
$$

The corresponding sum of all irreducible graphs $\sigma$ in the case of the locator expansion is called the locator; similarly to Eq. (37), we have

$$
\langle U\rangle=H_{0}+H_{0} \sigma\langle U\rangle \text {. }
$$

From Eqs. (42) and (44) it immediately follows that in terms of the locator the Dyson equation and the expression for $\langle G\rangle$ are

$$
\begin{aligned}
& \langle G\rangle=\sigma+\sigma H_{0}\langle G\rangle, \\
& \langle G\rangle=\left(\sigma^{-1}-H_{0}\right)^{-1},
\end{aligned}
$$

respectively. The relationship between the locator $\sigma$ and the self-energy $\Sigma$, according to Eqs. (38) and (46), is

$$
\sigma=(E-\Sigma)^{-1}
$$

\section{Renormalization of one-electron potential}

We now renormalize the potential $V$ subtracting a configuration-independent, sitediagonal operator $S$ and adding it to the unperturbed Hamiltonian $H_{0}$ :

$$
H=H_{0}+V=\left(H_{0}+S\right)+(V-S)=\tilde{H}+\tilde{V} .
$$

If one defines the unperturbed Green's function with respect to the renormalized Hamiltonian $\tilde{H}$,

$$
\tilde{G}=(E-\tilde{H})^{-1},
$$

then the Green's functions $G$ and $\tilde{G}$ are related by the Dyson equation analogous to Eq. (35),

$$
G=\tilde{G}+\tilde{G} \tilde{V} G
$$

As follows from Eqs. (38) and (48),

$$
\langle G\rangle=(E-\tilde{H}-\tilde{\Sigma})^{-1}
$$

where $\tilde{\Sigma}$ relates to $\tilde{H}$ and $\tilde{V}$ in the same way as $\Sigma$ to $H_{0}$ and $V$, and

$$
\Sigma=S+\tilde{\Sigma}
$$

The operator $S$ may be regarded as an initial approximation for the exact self-energy $\Sigma$.

The next step is to introduce the total scattering operator $T$ and express the exact Green's function $G$ and the self-energy $\Sigma$ in terms of $T$. The total scattering operator is defined by the relation

$$
T=\tilde{V}+\tilde{V} \tilde{G} T
$$

which gives

$$
T=\left(\tilde{V}^{-1}-\tilde{G}\right)^{-1} .
$$

Excluding $\tilde{V}$ from Eqs. (50) and (54) and averaging the result over realizations of the random potential, we get the relation between $\langle G\rangle$ and $\langle T\rangle$ :

$$
\langle G\rangle=\tilde{G}+\tilde{G}\langle T\rangle \tilde{G}
$$


Taking into account the relation (38) between the averaged Green's function and the self-energy and using Eq. (55), we finally obtain

$$
\Sigma=S+\left(\langle T\rangle^{-1}+\tilde{G}\right)^{-1} .
$$

Thus, having calculated the averaged total scattering operator $\langle T\rangle$ one can determine the averaged Green's function $\langle G\rangle$ and the self-energy $\Sigma$ according to Eqs. (55) and (56). The problem of the description of the disordered alloy is therefore reduced to the problem of finding reasonable approximate expression for the operator $\langle T\rangle$. The operator $T$ can be expressed in terms of operators describing scattering on individual atomic potentials $V_{i}$. To do this, it is convenient to decompose the Green's function $\tilde{G}$ into two parts which are diagonal and off-diagonal in the site representation, respectively:

$$
\tilde{G}=\tilde{G}_{d}+\tilde{G}_{o d}
$$

Inserting this equation into Eq. (54), we have after some straightforward algebra,

$$
T=\left(t^{-1}-\tilde{G}_{o d}\right)^{-1}
$$

where the operator

$$
t=\left(\tilde{V}^{-1}-\tilde{G}_{d}\right)^{-1}
$$

is, similarly to the potential $V$, site-diagonal and represents a sum of individual scattering operators $t_{i}$ corresponding to atomic potentials.

\section{Single-site approximations}

At any level of approximation there exist two main approaches to the problem of calculation of the averaged total scattering operator $\langle T\rangle$ and, consequently, the averaged Green's function $\langle G\rangle$ and the self-energy $\Sigma$. First, it is possible to choose the operator $S$ from the very beginning, and then calculate these three quantities using Eqs. (54)-(56); this is the non-self-consistent scheme. In most cases the VCA choice $S=\langle V\rangle$ is used. Second, one can consider $S$ as an operator variable and, noticing that the scattering operator $T$ is a function of $S$, determine the latter as a solution of the equation

$$
\langle T[S]\rangle=0 .
$$

This equation gives, according to Eqs. (55) and (56),

$$
\begin{aligned}
\langle G\rangle & =\tilde{G}, \\
\Sigma & =S,
\end{aligned}
$$

and such an approach is called self-consistent. To make the calculation of the averaged total scattering operator practically possible, the following decoupling of the configurationally-averaged Eq. (58), called the SSA, is usually adopted:

$$
\langle T\rangle=\left\langle\left(t^{-1}-\tilde{G}_{o d}\right)^{-1}\right\rangle \stackrel{S S A}{\longrightarrow}\left(\langle t\rangle^{-1}-\tilde{G}_{o d}\right)^{-1} .
$$

In the framework of the SSA Eq. (56) for the self-energy takes the form

$$
\Sigma^{S S A}=S+\left(\langle t\rangle^{-1}+\tilde{G}_{d}\right)^{-1}
$$

which means that in this approximation the self-energy is diagonal in the site representation. Consider now the two approaches in combination with the SSA. The nonself-consistent one, as is seen from Eqs. (59) and (64), gives the self-energy as a sum of 
the operator $S$ and the effective scattering potential $\tilde{V}_{\text {eff }}$ corresponding to the average scattering operator $\langle t\rangle$,

$$
\tilde{V}_{e f f}=\left(\langle t\rangle^{-1}+\tilde{G}_{d}\right)^{-1}
$$

accordingly, this method of calculation is known as the ATA. The application of the SSA to the self-consistent scheme (Eq. (60)) gives the following equation for the evaluation of the self-energy:

$$
\langle t[S]\rangle=0
$$

This equation determines the CPA.

\section{EXPRESSION FOR SHORT-RANGE ORDER DIFFUSE INTENSITY}

We now derive a formally exact expression for the SRO part $\alpha(\mathbf{k})$ of the diffusescattering intensity. A key observation here is that the spin PCF (22) satisfies the Dyson equation, ${ }^{8}$

$$
G=\sigma+\sigma \Delta G
$$

where $\Delta=\beta J$ and, like in the previous section, matrix notations are used. Here $\sigma$ is again the sum of all irreducible graphs in the diagrammatic expansion for $G$, but irreducibility is now defined with respect to the interaction-to-temperature ratio $\Delta$. Eq. (67) has the same form as the Dyson equation (45) for the Green's function of electrons (32) averaged over alloy configurations. From Eq. (67) it follows that

$$
G=\left(\sigma^{-1}-\Delta\right)^{-1}
$$

This expression for the spin PCF is analogous to Eq. (46).

The irreducible part $\sigma$ of the PCF $G$ is sometimes called the self-energy. ${ }^{8}$ However, to maintain the analogy with the electronic theory of alloys (i.e., with the terminology of the previous section) we will refer to this quantity as the locator, and reserve this term for another object, defining the PCF self-energy $\Sigma$ by the relation similar to Eq. (47):

$$
\sigma=-\Sigma^{-1}
$$

In terms of $\Sigma$ Eq. (68) becomes

$$
G=(-\Sigma-\Delta)^{-1}
$$

We also introduce the locator $\tilde{\sigma}$ and the self-energy $\tilde{\Sigma}$ for the occupation-number PCFs $G^{\alpha \beta}$ (Eq. (21)):

$$
\begin{aligned}
\tilde{\sigma} & =\frac{1}{4} \sigma, \\
\tilde{\Sigma} & =4 \Sigma .
\end{aligned}
$$

Then matrices $\tilde{\sigma}$ and $\tilde{\Sigma}$ are related by the same Eq. (69),

$$
\tilde{\sigma}=-\tilde{\Sigma}^{-1}
$$

and we have

$$
G^{A A}=G^{B B}=-G^{A B}=c(1-c) \alpha=(-\tilde{\Sigma}+2 \beta V)^{-1}
$$


Written in k-representation, this equation leads to the following expression for the diffuse-scattering intensity: ${ }^{12,13}$

$$
\alpha(\mathbf{k})=\frac{1}{c(1-c)[-\tilde{\Sigma}(\mathbf{k})+2 \beta V(\mathbf{k})]} .
$$

The central quantity of interest for us here is the PCF self-energy $\tilde{\Sigma}(\mathbf{k})$; apart from the wavevector, it depends also on two other variables, temperature and concentration. Later on we will be focusing on existing approximations for the PCF self-energy.

\section{SELF-ENERGY AND THERMODYNAMICS}

In the last section the Dyson equation (67) and the related expression (70) for the spin PCF were simply postulated. However, it would be useful to know how Eq. (70) could be derived and how the self-energy is related to the thermodynamics of the system.

\section{Variational formulation of statistical mechanics}

As was mentioned before, the main task of the statistical-mechanical treatment is to calculate the partition function (6) of a system described by the Hamiltonian $H$. The Hamiltonian usually is a linear combination,

$$
H=\sum_{n} x_{n} a_{n}
$$

of some operators $a_{n}$ with coefficients $x_{n}$. Variables $\alpha_{n}$ conjugated to the parameters $x_{n}$ are defined as averages (20) of the operators $a_{n}$,

$$
\alpha_{n}=\left\langle a_{n}\right\rangle=\frac{\partial F}{\partial x_{n}}
$$

where

$$
F=-k_{B} T \ln Z
$$

is the free energy of the system, and the second equation in (77) comes from the definition (6) and Eq. (76). Our aim now is the calculation of the averages $\alpha_{n}$ and the free energy $F$ as functions of the parameters $x_{n}$. This problem can be formulated as a variational one, if the Legendre transform $\Gamma(\alpha)$ of the free energy $F(x)$ is introduced: ${ }^{14,15}$

$$
\Gamma(\alpha)=F(x(\alpha))-\sum_{n} \alpha_{n} x_{n}(\alpha)
$$

Here the averages $\alpha_{n}$ are the independent variables, and $x_{n}(\alpha)$ are solutions of Eqs. (77). Differentiating $\Gamma(\alpha)$ with respect to $\alpha_{n}$ and using Eqs. (77), we get

$$
\frac{\partial \Gamma}{\partial \alpha_{n}}=\sum_{m} \frac{\partial F}{\partial x_{m}} \frac{\partial x_{m}}{\partial \alpha_{n}}-\sum_{m}\left(\frac{\partial \alpha_{m}}{\partial \alpha_{n}} x_{m}+\alpha_{m} \frac{\partial x_{m}}{\partial \alpha_{n}}\right)=-x_{n} .
$$

Finally, introducing another function $\Phi(x, \alpha)$,

$$
\Phi(x, \alpha)=\Gamma(\alpha)+\sum_{n} \alpha_{n} x_{n}
$$


which depends on both $\alpha_{n}$ and $x_{n}$, we find from Eq. (80) that it is stationary with respect to variations of $\alpha_{n}$ at fixed $x_{n}$ :

$$
\frac{\partial \Phi}{\partial \alpha_{n}}=0
$$

At the stationary point $\alpha_{n}=\alpha_{n}(x)$, where $\alpha_{n}(x)$ are solutions of Eqs. (80), $\Phi(x, \alpha)$ coincides with the free energy $F(x)$ :

$$
\Phi(x, \alpha(x))=F(x) .
$$

Function $\Phi(x, \alpha)$ is usually called the variational free energy. Noting that the internal energy is the statistical average (20) of the Hamiltonian, $E=\langle H\rangle, \Phi(x, \alpha)$ can be written in the standard thermodynamic form:

$$
\begin{aligned}
\Phi(x, \alpha) & =E(x, \alpha)-T S(\alpha) \\
E(x, \alpha) & =\sum_{n} x_{n} \alpha_{n} \\
S(\alpha) & =-\beta \Gamma(\alpha)
\end{aligned}
$$

where $E(x, \alpha)$ and $S(\alpha)$ are the variational internal energy and configurational entropy, respectively. Eq. (82) now becomes

$$
T \frac{\partial S}{\partial \alpha_{n}}=x_{n}
$$

From Eqs. (77) and (80) it follows that

$$
\sum_{l} \frac{\partial^{2} \Gamma}{\partial \alpha_{n} \partial \alpha_{l}} \frac{\partial^{2} F}{\partial x_{l} \partial x_{m}}=-\sum_{l} \frac{\partial x_{n}}{\partial \alpha_{l}} \frac{\partial \alpha_{l}}{\partial x_{m}}=-\frac{\partial x_{n}}{\partial x_{m}}=-\delta_{n m} .
$$

Eq. (88) shows that matrices of second derivatives of the free energy $F(x)$ and its Legendre transform $\Gamma(\alpha)$ (or the variational free energy $\Phi(x, \alpha)$ which differs from $\Gamma(\alpha)$ only by the bilinear term $\left.\sum_{n} x_{n} \alpha_{n}\right)$ are mutually inverse up to a sign.

\section{First Legendre transformation for the Ising model}

We will now apply the general technique of the Legendre transformations outlined above to the particular case of the Ising model in the inhomogeneous magnetic field. The Hamiltonian of the model is a straightforward generalization of Eq. (13):

$$
H=-\frac{1}{2} \sum_{i j} J_{i j} s_{i} s_{j}-\sum_{i} h_{i} s_{i} .
$$

From comparison of Eqs. (76) and (89) it follows that the latter contains two kinds of operators $a_{n}$ - single spin variables $s_{i}$ and products $s_{i} s_{j}$ of two spin variables. Corresponding parameters $x_{n}$, except for sign, are $h_{i}$ and $J_{i j}$, and variables $\alpha_{n}$ conjugated to these parameters are $\left\langle s_{i}\right\rangle=m_{i}$ (Eq. (18)) and $\left\langle s_{i} s_{j}\right\rangle=m_{i} m_{j}+G_{i j}$ (Eq. (22)). In the general case considered before the Legendre transformation was carried out with respect to all parameters $x_{n}$; as a result, all conjugated variables $\alpha_{n}$ were calculated using the variational principle. Here, however, this approach will be applied only to the magnetic field $h_{i}$, and only the magnetization $m_{i}$ will be calculated by means of the variational procedure. The resulting partial transformation is called the first Legendre 
transformation. ${ }^{15-17}$ In this case $h_{i}$ plays the role of $x_{n}$, and comparison with Eq. (76) shows that $a_{n}$ corresponds to $-s_{i}$. Eqs. (77), (79)-(83) and (88) now become

$$
\begin{aligned}
\Gamma(m, J) & =F(h(m), J)+\sum_{i} m_{i} h_{i}(m), \\
\Phi(h, m ; J) & =\Gamma(m, J)-\sum_{i} m_{i} h_{i}, \\
\frac{\partial F}{\partial h_{i}} & =-m_{i}, \\
\frac{\partial \Gamma}{\partial m_{i}} & =h_{i}, \\
\frac{\partial \Phi}{\partial m_{i}} & =0, \\
\Phi(h, m(h) ; J) & =F(h, J), \\
\sum_{k} \frac{\partial^{2} \Gamma}{\partial m_{i} \partial m_{k}} \frac{\partial^{2} F}{\partial h_{k} \partial h_{j}} & =\sum_{k} \frac{\partial^{2} \Phi}{\partial m_{i} \partial m_{k}} \frac{\partial^{2} F}{\partial h_{k} \partial h_{j}}=-\delta_{i j},
\end{aligned}
$$

where, in the same manner as earlier, $h_{i}(m)$ and $m_{i}(h)$ are solutions of Eqs. (92) and (93), respectively.

\section{Derivation of the Dyson equation and meaning of the self-energy}

First of all, we note that from the definitions of the partition function, the statistical average, the PCF and the free energy (Eqs. (61), (20), (22) and (78)) it follows that for the Hamiltonian (89)

$$
\frac{\partial^{2} F}{\partial h_{i} \partial h_{j}}=-\beta G_{i j} \text {. }
$$

Then, combining this equation with Eq. (96), we obtain

$$
G_{i j}=k_{B} T\left(\frac{\partial^{2} \Phi}{\partial m \partial m}\right)_{i j}^{-1}
$$

This notation means that the real-space matrix element of the PCF is proportional to the corresponding matrix element of the inverse of the matrix whose matrix elements are second derivatives $\partial^{2} \Phi / \partial m_{i} \partial m_{j}$ of the variational free energy with respect to the corresponding magnetizations.

The variational free energy can always be written as a sum of its mean-field (BraggWilliams) part and the non-mean-field correction:

$$
\begin{aligned}
\Phi & =\Phi^{M F A}+\delta \Phi, \\
\Phi^{M F A} & =E^{M F A}-T S^{M F A} .
\end{aligned}
$$

The expressions for the mean-field internal energy $E^{M F A}$ and the configurational entropy $S^{M F A}$ are well-known: ${ }^{1-6}$

$$
\begin{aligned}
E^{M F A} & =-\frac{1}{2} \sum_{i j} J_{i j} m_{i} m_{j}-\sum_{i} h_{i} m_{i}, \\
S^{M F A} & =-k_{B} \sum_{i}\left(\frac{1+m_{i}}{2} \ln \frac{1+m_{i}}{2}+\frac{1-m_{i}}{2} \ln \frac{1-m_{i}}{2}\right) .
\end{aligned}
$$


Substituting Eqs. (99)-(102) into Eq. (98) and noticing that

$$
\frac{\partial^{2} E^{M F A}}{\partial m_{i} \partial m_{j}}=-J_{i j}
$$

we recover the result (770) for the PCF (and, therefore, the Dyson equation (67)) with the following expression for the self-energy:

$$
\Sigma_{i j}=\frac{\partial^{2} S^{M F A}}{\partial m_{i} \partial m_{j}}-\beta \frac{\partial^{2}(\delta \Phi)}{\partial m_{i} \partial m_{j}} .
$$

It is seen that the self-energy is the sum of the second derivatives, with respect to the magnetizations, of the two terms contributing to the expression for the variational free energy $\Phi$ : the mean-field configurational entropy and the non-mean-field part of $\Phi$. Noting further that

$$
\frac{\partial^{2} S^{M F A}}{\partial m_{i} \partial m_{j}}=-\frac{\delta_{i j}}{1-m_{i}^{2}},
$$

we finally obtain

$$
\Sigma_{i j}=-\frac{\delta_{i j}}{1-m_{i}^{2}}-\beta \frac{\partial^{2}(\delta \Phi)}{\partial m_{i} \partial m_{j}} .
$$

The first term in this expression is diagonal in the direct space. This means that, back to the homogeneous case in which all the lattice sites are equivalent, this term is $\mathbf{k}$-independent in the reciprocal space, and all the wavevector dependence of the selfenergy comes from the second term, i.e., is the result of the non-mean-field corrections to the MFA variational free energy.

\section{APPROXIMATIONS FOR THE SELF-ENERGY}

In this section our attention will be focused on the formally exact result (75) for the SRO diffuse intensity $\alpha(\mathbf{k})$, which is the Fourier transform of the Warren-Cowley SRO parameters $\alpha_{i j}$ (24). Available theories of SRO will now be considered, in the light of the structure of Eq. (75), as different approximations for the self-energy $\tilde{\Sigma}$.

\section{Random-phase (Krivoglaz-Clapp-Moss) approximation}

The simplest and by far the most popular theory of SRO is the RPA (or, in the alloy language, the KCM approximation): ${ }^{, 18,19}$

$$
\alpha^{R P A}(\mathbf{k})=\frac{1}{1+2 c(1-c) \beta V(\mathbf{k})} .
$$

Eq. (107) is usually derived using mean-field-like arguments. Comparing Eqs. (75) and (107), we see that

$$
\tilde{\Sigma}^{R P A}(\mathbf{k})=-\frac{1}{c(1-c)}
$$

The RPA self-energy (108) is thus wavevector- and temperature-independent; it depends only on alloy composition. Returning via Eqs. (19) and (72) to the magnetic language used in the previous section, we conclude that the RPA for the self-energy corresponds precisely to neglecting the second term in the right-hand side of Eq. (106). The non-mean-field contribution to the self-energy is therefore ignored in the RPA, 
and the self-energy in the direct space is simply the second derivative of the mean-field configurational entropy with respect to the magnetization:

$$
\Sigma_{i j}^{R P A}=\frac{\partial^{2} S^{M F A}}{\partial m_{i} \partial m_{j}}=-\frac{\delta_{i j}}{1-m_{i}^{2}} .
$$

From the point of view of the terminology used in the electronic theory of disordered alloys, the RPA for the self-energy could be referred to as the SSA; indeed, both the RPA (Eq. (104)) and the SSA (Eq. (64)) self-energies are diagonal in the site representation. The RPA resembles the non-self-consistent SSA, i.e., the ATA, in the sense that both approximations define the corresponding self-energies explicitly.

The RPA reduces to the well-known Ornstein-Zernike description of correlations, ${ }^{7}$ when only those wavevectors which are close to the position $\mathbf{k}_{0}$ of the absolute minimum of the interaction $V(\mathbf{k})$ are considered (this approximation corresponds to the long-wave limit in the case of ferromagnetic ordering). Let us expand $V(\mathbf{k})$ in powers of $\mathbf{q}=\mathbf{k}-\mathbf{k}_{0}$ and retain only the lowest-order (quadratic) term,

$$
V(\mathbf{k}) \approx V\left(\mathbf{k}_{0}\right)+\frac{1}{2} \sum_{i j} g_{i j} q_{i} q_{j}
$$

where $g$ is the $3 \times 3$-matrix of second derivatives of $V(\mathbf{k})$ at $\mathbf{k}_{0}$. We will take only the simplest example of cubic symmetry, when $g_{i j}=g \delta_{i j}$; in this case $\sum_{i j} g_{i j} q_{i} q_{j}=g q^{2}$, where $q \equiv|\mathbf{q}|$. Substituting the result into Eq. (107), we obtain

$$
\alpha^{R P A}(\mathbf{k})=\frac{k_{B} T}{k_{B}\left(T-T_{c}\right)+c(1-c) g q^{2}},
$$

where it is taken into account that the mean-field result for the instability temperature $T_{c}$ is

$$
T_{c}=2 c(1-c)\left|V\left(\mathbf{k}_{0}\right)\right| / k_{B} ;
$$

at the position of the absolute minimum the interaction value is negative, since the average of the interaction over the Brillouin zone is zero (see Eq. (116) below). In real space we get asymptotically (i.e., at large distances)

$$
\alpha_{i j}^{R P A} \propto \frac{1}{r_{i j}} \exp \left(-i \mathbf{k}_{0} \mathbf{r}_{i j}-r_{i j} / \xi\right)
$$

where $r_{i j} \equiv\left|\mathbf{r}_{i j}\right|$, and the correlation length $\xi$ is

$$
\xi=\sqrt{\frac{c(1-c) g}{k_{B}\left(T-T_{c}\right)}} .
$$

Eqs. (111)-(114) represent the Ornstein-Zernike result for the PCF.

However, the RPA expression (107) has a serious disadvantage: it is unable to satisfy the sum rule (31). Using the identity

$$
\frac{1}{1+2 c(1-c) \beta V(\mathbf{k})}=1-2 c(1-c) \beta V(\mathbf{k})+\frac{4 c^{2}(1-c)^{2} \beta^{2} V^{2}(\mathbf{k})}{1+2 c(1-c) \beta V(\mathbf{k})},
$$

it can be shown that the RPA formula (107) always leads to the overestimation of the value of the integral over the Brillouin zone (31). Since

$$
\frac{1}{\Omega} \int d \mathbf{k} V(\mathbf{k})=V_{i i}=0
$$


from Eq. (115) it follows that

$$
\frac{1}{\Omega} \int d \mathbf{k} \alpha^{R P A}(\mathbf{k})=1+\frac{1}{\Omega} \int d \mathbf{k} \frac{4 c^{2}(1-c)^{2} \beta^{2} V^{2}(\mathbf{k})}{1+2 c(1-c) \beta V(\mathbf{k})},
$$

and, therefore,

$$
\frac{1}{\Omega} \int d \mathbf{k} \alpha^{R P A}(\mathbf{k})>1
$$

This integral is close to unity only at sufficiently high temperatures. As temperature decreases, the deviation from the value prescribed by the sum rule becomes more and more significant, and the integral finally diverges at the instability point. ${ }^{20,21}$

\section{Spherical model}

Another analytical expression for $\alpha(\mathbf{k})$ is given by the SM,,$^{6,19,22-24}$ also known as the Onsager cavity field theory, ${ }^{25,26}$

$$
\alpha^{S M}(\mathbf{k})=\frac{1}{c(1-c)[-\tilde{\Sigma} S M+2 \beta V(\mathbf{k})]},
$$

where $\tilde{\Sigma}^{S M}$ is, at fixed temperature and concentration, a number determined from the sum rule (31). Therefore, the sum rule is satisfied in the SM by definition, contrary to the case of the RPA. From the definition of the SM it also follows that the selfenergy depends not only on concentration, like its RPA counterpart (108), but also on temperature. Nevertheless, the SM self-energy is still wavevector-independent. The explicit expression for $\tilde{\Sigma}^{S M}$ can be derived from the sum rule (31):

$$
\begin{aligned}
\tilde{\Sigma}^{S M} & =\tilde{\Sigma}^{R P A}+\delta \tilde{\Sigma} \\
\delta \tilde{\Sigma} & =2 \beta \sum_{j} \alpha_{i j} V_{i j}=\frac{2 \beta}{\Omega} \int d \mathbf{k} \alpha(\mathbf{k}) V(\mathbf{k}) .
\end{aligned}
$$

Similarly to the RPA, the SM is analogous to the SSA, since the SM self-energy is diagonal in the direct space. However, the SM is rather the self-consistent SSA, like the CPA, because the sum rule here plays the role of the CPA self-consistency condition. In fact, the sum rule is the self-consistency condition: it simply means that the diagonal matrix elements of the approximate and exact PCFs are the same. More surprisingly, it was shown ${ }^{27,28}$ that both the CPA and the SM could be obtained by summation of the same sets of diagrams in the corresponding perturbation expansions. We can conclude, therefore, that the SM is the CPA for the Ising model.

\section{Cluster variation method}

The $\mathrm{CVM}^{29}$ is at present the standard technique for quantitative calculation of thermodynamic properties of alloys. It is discussed in great detail in almost every book or review on the subject. ${ }^{1-3,6}$ We do not attempt to do this here; instead, we will consider only those features of the CVM which are relevant to our discussion, without going into technical aspects of the method.

The CVM is essentially a procedure which allows us to derive an approximate expression for the variational configurational entropy $S(\alpha)$ of the system. The CVM entropy is a function of probabilities of various atomic configurations on lattice clusters which belong to the so-called basic, or maximal, cluster. A particular CVM approximation for $S(\alpha)$ is therefore defined by the choice of the basic cluster. Combined with 
the variational internal energy $E(x, \alpha)$, the CVM configurational entropy gives the expression (84) for the variational free energy $\Phi(x, \alpha)$. The operators $a_{n}$ in the alloy Hamiltonian are products of the spin variables or of the occupation numbers, and the averages $\alpha_{n}$ (Eq. (77)) are thus related to, or coincide with, cluster probabilities entering the expression for the CVM configurational entropy. The variational free energy $\Phi$ is then minimized with respect to all cluster probabilities, taking into account various self-consistency constraints.

The self-energy obtained in the framework of the CVM depends, in general, on all three parameters - temperature, concentration and wavevector. The problem with the CVM, however, is that this method is intrinsically numerical and does not lead to analytical approximations for pair correlations. The reason for this is that in most cases the number of cluster variables used to get a reasonably accurate formula for the configurational entropy is far too large. From the point of view of the general technique of the Legendre transformations, the CVM corresponds to the high-order transformation with respect to all coefficients $x_{n}$ conjugated to cluster variables which are involved in the expression for the variational configurational entropy $S(\alpha)$. Therefore, as far as SRO is concerned, general Eq. (38) is still valid, as is the Ising model-specific Eq. (97). However, in practical sense this case is very different from that of the first Legendre transformation. In the latter, the inversion of the matrix $\partial^{2} \Phi / \partial \alpha \partial \alpha$ is carried out trivially using the Fourier transformation. In the CVM this object in the reciprocal space is still a sufficiently large matrix, and in all realistic situations it is necessary to resort to numerics. Analytical formulae were obtained only for such simple model cases as the pair (also known as quasichemical, or Fowler-Guggenheim, or Bethe) approximation, or the square (Kramers-Wannier) approximation for the nearest-neighbour Ising model on the square lattice. ${ }^{2,20}$ Besides, the CVM suffers from the same drawback as the RPA, though to a lesser extent: the integrated intensity is not conserved, and its behavior with temperature is similar to the case of the RPA. ${ }^{2,20}$

\section{High-temperature expansion}

As we have seen, the RPA and the SM are the analogs of the SSAs in the electronic theory of disordered alloys and thus fail to take account of the wavevector dependence of the self-energy. On the other hand, the CVM leads to the k-dependent self-energy, but loses the simplicity of the former two approximations by not providing analytical expressions for correlations. At the semiquantitative level of the RPA this problem can be cured by using the HTE for the self-energy. ${ }^{30}$

To do this, we return to Eq. (104) which, in combination with Eq. (109), can be written as

$$
\begin{aligned}
\Sigma & =\Sigma^{R P A}+\delta \Sigma, \\
\delta \Sigma_{i j} & =-\beta \frac{\partial^{2}(\delta \Phi)}{\partial m_{i} \partial m_{j}} .
\end{aligned}
$$

It is known that the RPA is exact to first order in $1 / T$ (i.e., in $\Delta$ ) $;^{2}$ it means that $\delta \Phi$ and $\delta \Sigma$ are of order $\Delta^{2}$. It is also known how to construct the first Legendre transformation $\Gamma$ (and, therefore, $\delta \Phi$ ) for the Ising model. ${ }^{15-17}$ Sorting all contributions to $\delta \Phi$ (diagrams) according to the number of lines $\Delta$, we obtain the HTE; first eight orders in $\Delta$ are available in the literature. ${ }^{17}$ For simplicity reasons, the discussion here is confined to two first orders,

$$
-\beta \delta \Phi=\frac{1}{4} \circlearrowright+\frac{1}{12} \bigodot+\frac{1}{6} \bigwedge+O\left(\Delta^{4}\right)
$$


where a line corresponds to $\Delta$, and a vertex with $n$ attached lines represents the function $u_{n}\left(m_{i}\right)$. For the diagrams in Eq. (124) $n$ is equal to either 2 or 3:

$$
\begin{aligned}
& u_{2}\left(m_{i}\right)=1-m_{i}^{2}, \\
& u_{3}\left(m_{i}\right)=-2 m_{i}\left(1-m_{i}^{2}\right) .
\end{aligned}
$$

The expressions for the diagonal and off-diagonal parts of $\delta \Sigma$ thus are, according to Eq. (123),

$$
\begin{aligned}
& \delta \Sigma_{i i}=\frac{1}{2} \bigcup+\frac{1}{6} Џ+\frac{1}{2} \searrow+O\left(\Delta^{4}\right) \\
& \delta \Sigma_{i j}=\frac{1}{2} \longmapsto+\frac{1}{6} \Longleftrightarrow+\leadsto+O\left(\Delta^{4}\right), i \neq j .
\end{aligned}
$$

Here a vertex with $n$ internal lines and $k$ external legs corresponds to the $k$ th derivative of the function $u_{n}(m)$. The corresponding analytical expressions have the form

$$
\begin{aligned}
\delta \Sigma_{i i}= & -\sum_{l}\left(1-m_{l}^{2}\right) \Delta_{i l}^{2}-4 m_{i} \sum_{l} m_{l}\left(1-m_{l}^{2}\right) \Delta_{i l}^{3} \\
& -\sum_{k l}\left(1-m_{k}^{2}\right)\left(1-m_{l}^{2}\right) \Delta_{i k} \Delta_{i l} \Delta_{k l}+O\left(\Delta^{4}\right), \\
\delta \Sigma_{i j}= & 2 m_{i} m_{j} \Delta_{i j}^{2}+\frac{2}{3}\left(1-3 m_{i}^{2}\right)\left(1-3 m_{j}^{2}\right) \Delta_{i j}^{3} \\
& +4 m_{i} m_{j} \Delta_{i j} \sum_{l}\left(1-m_{l}^{2}\right) \Delta_{i l} \Delta_{j l}+O\left(\Delta^{4}\right), \quad i \neq j .
\end{aligned}
$$

In the homogeneous case $m_{i}=m$ for all sites $i$. Defining constants

$$
a_{1}=\sum_{l} J_{i l}^{2}, \quad a_{2}=\sum_{l} J_{i l}^{3}, \quad a_{3}=\sum_{k l} J_{i k} J_{i l} J_{k l}
$$

and lattice functions

$$
\left(f_{1}\right)_{i j}=J_{i j}^{2}, \quad\left(f_{2}\right)_{i j}=J_{i j}^{3}, \quad\left(f_{3}\right)_{i j}=J_{i j} \sum_{l} J_{i l} J_{j l},
$$

which depend only on the interaction $J$, one can finally write the expression for $\delta \Sigma$ in the k-space:

$$
\begin{aligned}
\delta \Sigma(\mathbf{k})= & -\left(1-m^{2}\right) \beta^{2} a_{1}+2 m^{2} \beta^{2} f_{1}(\mathbf{k})-4 m^{2}\left(1-m^{2}\right) \beta^{3} a_{2} \\
& -\left(1-m^{2}\right)^{2} \beta^{3} a_{3}+\frac{2}{3}\left(1-3 m^{2}\right)^{2} \beta^{3} f_{2}(\mathbf{k}) \\
& +4 m^{2}\left(1-m^{2}\right) \beta^{3} f_{3}(\mathbf{k})+O\left(\beta^{4}\right) .
\end{aligned}
$$

In alloy notations this result reads

$$
\begin{aligned}
\delta \tilde{\Sigma}(\mathbf{k})= & -4 c(1-c) \beta^{2} \tilde{a}_{1}+2(1-2 c)^{2} \beta^{2} \tilde{f}_{1}(\mathbf{k})+8 c(1-c)(1-2 c)^{2} \beta^{3} \tilde{a}_{2} \\
& +8 c^{2}(1-c)^{2} \beta^{3} \tilde{a}_{3}-\frac{4}{3}[1-6 c(1-c)]^{2} \beta^{3} \tilde{f}_{2}(\mathbf{k}) \\
& -8 c(1-c)(1-2 c)^{2} \beta^{3} \tilde{f}_{3}(\mathbf{k})+O\left(\beta^{4}\right)
\end{aligned}
$$

where

$$
\begin{aligned}
\tilde{a}_{1} & =\sum_{l} V_{i l}^{2}, \quad \tilde{a}_{2}=\sum_{l} V_{i l}^{3}, \quad \tilde{a}_{3}=\sum_{k l} V_{i k} V_{i l} V_{k l}, \\
\left(\tilde{f}_{1}\right)_{i j} & =V_{i j}^{2}, \quad\left(\tilde{f}_{2}\right)_{i j}=V_{i j}^{3}, \quad\left(\tilde{f}_{3}\right)_{i j}=V_{i j} \sum_{l} V_{i l} V_{j l} .
\end{aligned}
$$




\section{Alpha- and gamma-expansions}

The approximate expressions (133), (134) for the self-energy given by the first orders of the HTE satisfy almost all requirements: they are analytical, relatively simple and take into account dependence on all relevant parameters, including the wavevector. However, the limits of applicability of the HTE are essentially the same as those of the RPA; the HTE is quantitatively correct only at reasonably high temperatures, as can be concluded already from the name of the expansion. What is needed, therefore, is an approximation which would combine all the advantages of the HTE self-energy with the applicability at moderate temperatures, including the range not far away from the transition or instability points.

The theory of SRO which will now be discussed ${ }^{12,13}$ is based on the fairly general procedure ${ }^{27}$ of self-consistent renormalization of the bare propagator $\Delta^{-1}$ in the functional-integral representation of the generating functional for correlation functions. The resulting expansion for the matrix elements of the self-energy is in powers of the matrix elements of the fully dressed propagator, which in the case of the Ising model coincides with the PCF (70). Two first non-zero orders of this expansion for the offdiagonal part of the self-energy were calculated, ${ }^{12,13}$

$$
\begin{aligned}
\Sigma_{i j} & =a G_{i j}^{2}+b G_{i j}^{3}+O\left(G^{4}\right), \quad i \neq j, \\
a & =\frac{2 m^{2}}{\left(1-m^{2}\right)^{4}} \\
b & =\frac{2\left[\left(1-3 m^{2}\right)^{2}-12 m^{4}\right]}{3\left(1-m^{2}\right)^{6}},
\end{aligned}
$$

in terms of the alloy variables

$$
\begin{aligned}
\tilde{\Sigma}_{i j} & =\tilde{a} \alpha_{i j}^{2}+\tilde{b} \alpha_{i j}^{3}+O\left(\alpha^{4}\right), \quad i \neq j \\
\tilde{a} & =\frac{(1-2 c)^{2}}{2[c(1-c)]^{2}}, \\
\tilde{b} & =\frac{[1-6 c(1-c)]^{2}-3(1-2 c)^{4}}{6[c(1-c)]^{3}} .
\end{aligned}
$$

Eq. (140) is the expansion in powers of the SRO parameters, and is therefore referred to as the $\mathrm{AE},{ }^{31}$ though initially it was obtained in the framework of the GEM. ${ }^{12,13}$ The difference between the AE and GEM is discussed below. In the two calculated orders the AE preserves the sum rule (31), and the expression for the diagonal part of the self-energy then comes from Eqs. (31) and (140), similarly to the case of the SM (Eqs. (120), (121)):

$$
\begin{aligned}
\tilde{\Sigma}_{i i} & =\tilde{\Sigma}^{R P A}+\delta \tilde{\Sigma}_{i i} \\
\delta \tilde{\Sigma}_{i i} & =2 \beta \sum_{j} \alpha_{i j} V_{i j}-\sum_{j(\neq i)}\left(\tilde{a} \alpha_{i j}^{3}+\tilde{b} \alpha_{i j}^{4}\right)+O\left(\alpha^{5}\right) .
\end{aligned}
$$

Note that the first term in Eq. (144) corresponds to the SM (cf. Eq. (121)) which is the zero-order approximation for both the AE and the GEM; in the SM the self-energy is diagonal $(\tilde{a}=\tilde{b}=0)$.

The difference between the GEM and the AE is in the way of selection of leading terms in the expansion (140). The GEM, originally proposed by $\operatorname{Tokar}^{27}$ and further 
developed by Tokar, Grishchenko and the author, ${ }^{12,13,32-35}$ is based on the assumption that correlations decrease rapidly with distance, and the GEM expansion parameter is

$$
\gamma=\exp (-1 / \xi)
$$

where $\xi$ is the dimensionless correlation length. The leading terms in the diagrammatic expansion for the self-energy are selected in the framework of the GEM according to the total length of all lines in the diagrams, where the line connecting lattice sites $i$ and $j$ represents $\alpha_{i j}$. For example, in the case of three Bravais lattices belonging to the cubic system taking into account several first terms of the perturbation theory leads to the result ${ }^{12,13}$

$$
\begin{aligned}
& \tilde{\Sigma}_{s}=\tilde{a} \alpha_{s}^{2}+\tilde{b} \alpha_{s}^{3}, \quad s=1, \\
& \tilde{\Sigma}_{s}=\tilde{a} \alpha_{s}^{2}, \quad s=2,3, \\
& \tilde{\Sigma}_{s}=0, \quad s>3,
\end{aligned}
$$

where subscript $s$ denotes the matrix elements corresponding to the $s$ th coordination shell. However, the GEM assumption about the rapid decay of correlations is not always valid; e.g., it is incorrect in the cases where distant interactions are essential. The AE abandons this assumption and uses instead the SRO parameters $\alpha_{i j}$ themselves as the expansion parameters; the leading terms are chosen according to the number of lines in the diagrams (i.e., the powers of $\alpha_{i j}$ ), since all $\alpha_{i j}$ are relatively small. A particular AE approximation is defined by neglecting higher-order terms and including only finite number of coordination shells in the AE expression (140) for the off-diagonal part of the self-energy. The GEM was successfully applied to both the direct and inverse problems of alloy diffuse scattering, ${ }^{12,13,36-38}$ leading to reliable results everywhere except in the vicinity of the instability point, while the $\mathrm{AE}$ was used in the analysis of some of the diffuse-scattering anomalies discussed in the next section. ${ }^{31,39}$

\section{ANOMALIES IN ALLOY DIFFUSE SCATTERING}

In this last section we will show how expression (75) for the intensity $\alpha(\mathbf{k})$ and, in particular, the wavevector dependence of the self-energy $\tilde{\Sigma}(\mathbf{k})$ lead to straightforward explanation of recently observed unusual features (anomalies) of diffuse scattering from disordered alloys and to prediction of some new effects.

\section{Temperature-dependent Fermi surface-induced peak splitting}

This curious effect (the temperature dependence of the splitting) was discovered in 1996 by Reichert, Moss and Liang ${ }^{40}$ in the first in situ experiment to resolve the fine structure of the equilibrium diffuse scattering intensity from the disordered $\mathrm{Cu}_{3} \mathrm{Au}$ alloy. The separation of the split maxima changed reversibly, increasing with temperature. The same behavior of the splitting was also found ${ }^{41}$ by analysing results of the MC simulations for the $\mathrm{Cu}_{0.856} \mathrm{Al}_{0.144}$ alloy. ${ }^{42}$ The fourfold splitting of the intensity peaks located at the (110) and equivalent positions (Figure 1) is attributed to the indirect interaction of atoms via conduction electrons in an alloy whose Fermi surface has flat portions; the effective interatomic pair interaction $V(\mathbf{k})$ itself has split minima in the reciprocal space, and their location is determined by the wavevector $2 \mathbf{k}_{F}$ spanning these flat portions of the Fermi surface. ${ }^{5,43}$ It is usually assumed that $V(\mathbf{k})$ is temperatureindependent. This assumption is justified at least as far as positions of the $V(\mathbf{k})$ minima 
are concerned, since the $2 \mathbf{k}_{F}$ value is unlikely to change over the considered temperature range. Besides, the $\mathrm{MC}$ calculations ${ }^{42}$ in which the increase of the splitting with temperature was found ${ }^{41}$ were carried out for the temperature-independent pair interaction parameters. The standard RPA (KCM) treatment (Eq. (107)) predicts that positions of the intensity peaks coincide with those of the corresponding minima of $V(\mathbf{k})$, and, therefore, the splitting does not depend on temperature.

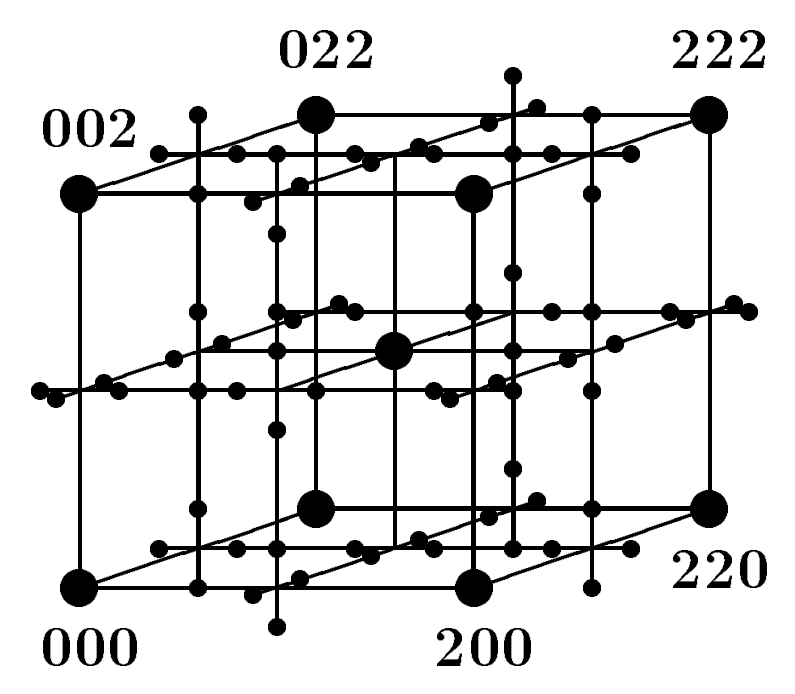

Figure 1. 3D reciprocal-space picture of scattering from the FCC alloys discussed in the text. Large dots represent the Bragg reflections. Characteristic crosses formed by small dots correspond to the split diffuse intensity peaks.

As will be demonstrated below, this phenomenon can be easily understood by employing the notion of the $\mathbf{k}$-dependent self-energy. ${ }^{31}$ Let us consider the $\alpha(\mathbf{k})$ profile along one of the lines containing split peaks, e.g., the (h10) line, and concentrate on the two peaks around the (110) position. The peak positions $k_{\alpha}(k$ is the deviation of the wavevector from the (110) position along the (h10) line) are determined by the condition $\partial \alpha / \partial k=0$ which gives

$$
2 \frac{\partial V}{\partial k}=T \frac{\partial \tilde{\Sigma}}{\partial k}
$$

Eq. (149) means that the k-dependence of $\tilde{\Sigma}$ leads to the shift $\delta k=k_{\alpha}-k_{V}$ of the peak position with respect to the position $k_{V}$ of the corresponding minimum of $V(\mathbf{k})$ (the latter is defined by the condition $\partial V / \partial k=0$ ). Furthermore, the right-hand side of Eq. (149) is a function of $T$, while its left-hand side is $T$-independent. The $\alpha(\mathbf{k})$ peaks will therefore change their positions with temperature. The "local" temperature behavior of the splitting is reflected in the sign of the derivative $\partial_{T} k_{I}$ which can be calculated by expanding the derivatives in Eq. (149) in powers of small changes of $T$ and $k_{I}$ and retaining only linear terms:

$$
\frac{\partial k_{\alpha}}{\partial T}=\left[\left(\frac{\partial \tilde{\Sigma}}{\partial k}+T \frac{\partial^{2} \tilde{\Sigma}}{\partial k \partial T}\right) /\left(2 \frac{\partial^{2} V}{\partial k^{2}}-T \frac{\partial^{2} \tilde{\Sigma}}{\partial k^{2}}\right)\right]_{k=k_{\alpha}} .
$$

Our aim now is to develop a kind of minimal, i.e., simplest possible, theory which would be able to describe essential features of the considered effect (and, apropos, two other 
anomalies discussed in this section). Interestingly, the approach formally rather similar to the Landau theory of second-order phase transitions ${ }^{7}$ could be used. Indeed, in the case of not very large splitting the expansion of $V(k)$ and $\tilde{\Sigma}(k)$ in powers of $k$ can be used. To describe the split minimum of $V(k)$, only the second- and fourth-order terms are necessary; since the (110) position serves as the origin, the expansions do not contain odd powers of $k$. We therefore assume that in the area of the splitting $V(k)$ and $\tilde{\Sigma}(k)$ have the following approximate form,

$$
\begin{aligned}
& V(k)=V(0)+\frac{1}{2} A_{V} k^{2}+\frac{1}{4} B_{V} k^{4}, \\
& \tilde{\Sigma}(k)=\tilde{\Sigma}(0)+\frac{1}{2} A_{\Sigma} k^{2}+\frac{1}{4} B_{\Sigma} k^{4},
\end{aligned}
$$

where $A_{V}<0, B_{V}>0$, the signs of $A_{\Sigma}$ and $B_{\Sigma}$ are arbitrary (there are no apparent restrictions on the behavior of the self-energy), and $k=0$ corresponds to the (110) position. The resulting inverse intensity $\alpha^{-1}(k)$ has exactly the form of the Landau free energy functional in the low-symmetry phase where the latter possesses a double minimum; this implies $2\left|A_{V}\right|+T A_{\Sigma}>0$ and $2 B_{V}-T B_{\Sigma}>0$. The wavevector $k$ plays the role of the order parameter. Substituting approximations (151) and (152) into general Eqs. (149) and (150), we get

$$
\begin{aligned}
k_{\alpha} & = \pm \sqrt{\frac{2\left|A_{V}\right|+T A_{\Sigma}}{2 B_{V}-T B_{\Sigma}}}, \\
k_{\alpha}^{-1} \frac{\partial k_{\alpha}}{\partial T} & =\frac{1}{2}\left[\frac{A_{\Sigma}+T \partial A_{\Sigma} / \partial T}{2\left|A_{V}\right|+T A_{\Sigma}}+\frac{B_{\Sigma}+T \partial B_{\Sigma} / \partial T}{2 B_{V}-T B_{\Sigma}}\right],
\end{aligned}
$$

while $k_{V}= \pm \sqrt{\left|A_{V}\right| / B_{V}}$. It is seen that the shifts of the two peaks and their temperature derivatives have opposite signs and the same absolute values. Eq. (154) clearly shows two scenarios for the temperature behavior of the splitting, depending on the sign of its right-hand side which can be either positive or negative. The first one is the increase of the splitting with temperature discussed above; this takes place when the right-hand side of Eq. (154) is negative. Apart from that, the theory predicts that the decrease of the splitting with increasing temperature is also possible. This regime corresponds to the case of positive right-hand side of Eq. (154), and such temperature dependence has not yet been observed experimentally. Thus, the behavior of the selfenergy determines whether the splitting increases or decreases with temperature. At high temperatures the correction $\delta \Sigma$ to the wavevector-independent $\Sigma^{R P A}$ (Eq. (122)), and, therefore, $A_{\Sigma}$ and $B_{\Sigma}$, are of order $\Delta^{2}$ (i.e., $T^{-2}$ ). From Eq. (153) it then follows that the absolute value of the shift $\delta k$ decreases as $T^{-1}$ with temperature, unless the corresponding coefficient identically vanishes.

\section{Coalescence of Fermi surface-related intensity peaks}

The analogy with the Landau theory of phase transitions, though rather formal, immediately leads to the following question: in the considered case, what would correspond to the transition point? The answer to this question is fairly obvious; there exists a possibility for the splitting to disappear at some point as temperature decreases, before the transition to the low-symmetry phase occurs. ${ }^{44}$ This anomaly was neither observed experimentally nor correctly described theoretically, though the coalescence of intensity peaks (unrelated to any Fermi surface effects) was found for the exactly solvable 1D Ising model ${ }^{45}$ and in the CVM calculations for the 2D ANNNI model. ${ }^{46}$ 
As we will show in this subsection, Eq. (75) provides clear understanding of how such effect takes place.

In the treatment given above it is, in fact, implicitly assumed that the wavevector dependence of the interaction term $2 \beta V(\mathbf{k})$ in Eq. (75) dominates, at least in the area of the splitting, i.e., near the (110) position. In this case the shape of the diffuse intensity closely follows that of $V(\mathbf{k})$; in particular, there exists one-to-one correspondence between the split minima of the interaction and the split intensity peaks. The variation of the self-energy with $\mathbf{k}$ in this part of the reciprocal space is comparatively weak, though qualitatively important for the description of the temperature-dependent splitting. This assumption is certainly correct at sufficiently high temperatures, where the RPA (KCM) approximation in which the self-energy is $\mathbf{k}$-independent works reasonably well. Meanwhile, as temperature starts to decrease, the correction to the RPA self-energy $\left(\propto T^{-2}\right)$ grows faster than the interaction term $2 \beta V(\mathbf{k})\left(\propto T^{-1}\right)$. We can then encounter a situation when the variations of $\tilde{\Sigma}(\mathbf{k})$ and $2 \beta V(\mathbf{k})$ with the wavevector are of the same order of magnitude. With temperature further decreasing, the wavevector dependence of the self-energy can even become dominant. In this regime positions of the diffuse intensity peaks would be determined by the maxima of $\tilde{\Sigma}(\mathbf{k})$.

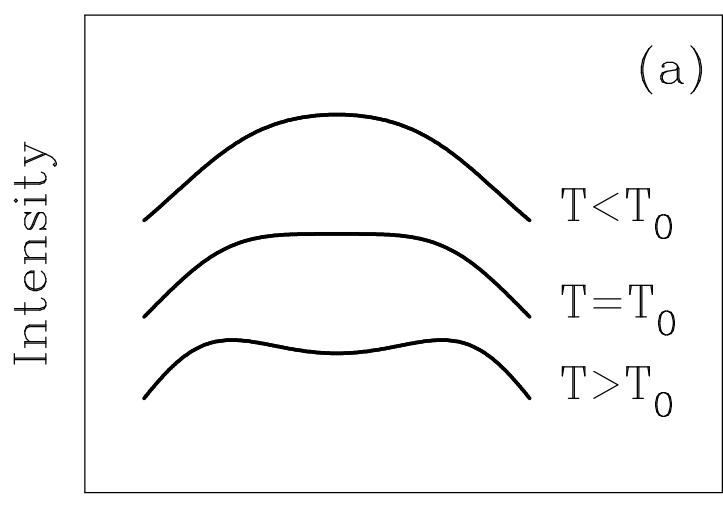

Wavevector

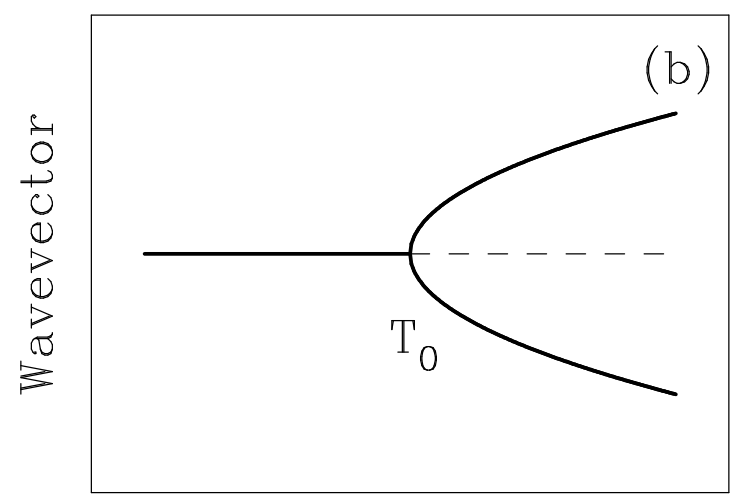

Temperature

Figure 2. Schematical temperature dependence of the intensity profile (a) and the peak positions (b) in the coalescence case.

The behavior of the self-energy in the k-space is, in general, qualitatively different from that of the interaction. In particular, there is no special reason to expect that the self-energy would have any extrema away from the special points. Let us assume that the self-energy does not have such extrema and that the variation of the self-energy in the reciprocal space becomes more and more important in comparison with the corresponding variation of $2 \beta V(\mathbf{k})$ as temperature decreases. Then the qualitative picture of the temperature behavior of the splitting is as follows. At high temperatures the self-energy is almost $\mathbf{k}$-independent, and the intensity peak positions deviate little from those of the corresponding minima of $V(\mathbf{k})$. As temperature decreases, the wavevector dependence of $\tilde{\Sigma}$ becomes more pronounced; the peak positions move farther away from the positions of $V(\mathbf{k})$ minima and towards those special points $\mathbf{k}_{0}$ at which $\tilde{\Sigma}(\mathbf{k})$ has maxima. Eventually, as temperature reaches certain value $T_{0}$, the intensity peaks coalesce at these special points and the splitting disappears (Figure 2).

The coalescence temperature $T_{0}$ can be found from the condition of vanishing second 
derivative $\alpha^{\prime \prime}=\partial^{2} \alpha / \partial k^{2}$ of the intensity with respect to the wavevector at $k_{0}$; the sign of this derivative controls the presence or absence of the splitting. At the special points all the first derivatives are equal to zero, and from Eq. (75) we obtain

$$
\alpha^{\prime \prime}\left(k_{0}\right)=c(1-c) \alpha^{2}\left(k_{0}\right)\left[\tilde{\Sigma}^{\prime \prime}\left(k_{0}\right)-2 \beta V^{\prime \prime}\left(k_{0}\right)\right] .
$$

Therefore, the splitting disappears when the second derivatives, or curvatures, of the self-energy and of the interaction term $2 \beta V(k)$ at the special point $k_{0}$ compensate each other, i.e., when

$$
\tilde{\Sigma}^{\prime \prime}\left(k_{0}\right)=2 \beta V^{\prime \prime}\left(k_{0}\right) .
$$

To analyse the behavior of the splitting close to the coalescence point, it is convenient to use the same Landau-type approach as in the previous case. In this temperature range the splitting above $T_{0}$ is small, and expansions (151) and (152) are valid. Substituting them into Eq. (75), we get

$$
\alpha^{-1}(k)=\alpha^{-1}(0)+\frac{1}{2} A k^{2}+\frac{1}{4} B k^{4},
$$

where second-order coefficient

$$
A=c(1-c)\left(-A_{\Sigma}+2 \beta A_{V}\right)
$$

vanishes at $T=T_{0}$ (see Eq. (156)), while the fourth-order coefficient $B$ remains positive at that temperature. We can then, at temperatures close to $T_{0}$, regard $A$ as linear in $T-T_{0}$ with a negative coefficient and $B$ as temperature-independent. Thus, the inverse intensity $\alpha^{-1}(k)$ behaves almost in the same way that the Landau free energy. The only difference here is that the role of temperature is reversed; $\alpha^{-1}(k)$ has a double minimum above the coalescence temperature $T_{0}$ and a single minimum below it. Therefore, at small positive values of $T-T_{0}$ the splitting increases with temperature as $\left(T-T_{0}\right)^{1 / 2}$. Contrary to the corresponding result of the genuine Landau theory, obtained bifurcation exponent is exact, since the intensity is an analytical function of the wavevector and can legitimately be expanded into the Taylor series. At higher temperatures behavior of the splitting changes, and sufficiently far away from $T_{0}$ it starts to approach the value dictated by the interaction $V(k)$.

\section{"Thermal" splitting of intensity peaks}

We have just considered the situation when the double-well profile of the interaction in the vicinity of a special point is compensated by the wavevector dependence of the self-energy which has a simple maximum at this position. The competing curvatures $2 \beta V^{\prime \prime}\left(k_{0}\right)$ and $\tilde{\Sigma}^{\prime \prime}\left(k_{0}\right)$ are both negative. As a result, the second derivative (155) of the intensity vanishes at some temperature, and the splitting induced by the Fermi surface effects disappears.

It is not very difficult to realize that another kind of curvature compensation is possible; this is the case in which both curvatures are positive. ${ }^{39}$ This is, perhaps, the most physically interesting situation: here the interaction with a single minimum produces the intensity peak with no fine structure at higher temperatures (in full accordance with the RPA-like considerations), but then, as temperature decreases and the wavevector dependence of the self-energy becomes more and more significant, the compensation takes place and the intensity peak splits. This is especially probable when the minimum of $V(k)$ is shallow (i.e., $V^{\prime \prime}\left(k_{0}\right)$ is small); in particular, in the limiting case of vanishing $V^{\prime \prime}\left(k_{0}\right)$ it is the curvature of $\tilde{\Sigma}(k)$ that controls the fine structure (single- vs. 
double-peak) of the maximum of $\alpha(k)$. The application of the Landau-type description gives essentially the same results as before. However, the coefficient in front of $T-T_{0}$ in A is now positive; the inverse intensity $\alpha^{-1}(k)$ has a single minimum above the splitting temperature $T_{0}$ and a double minimum below this point, and the splitting increases as $\left(T_{0}-T\right)^{1 / 2}$ with decreasing temperature at small negative $T-T_{0}$ values (Figure 3 ) . The bifurcation exponent is again exact, for the reasons mentioned above.

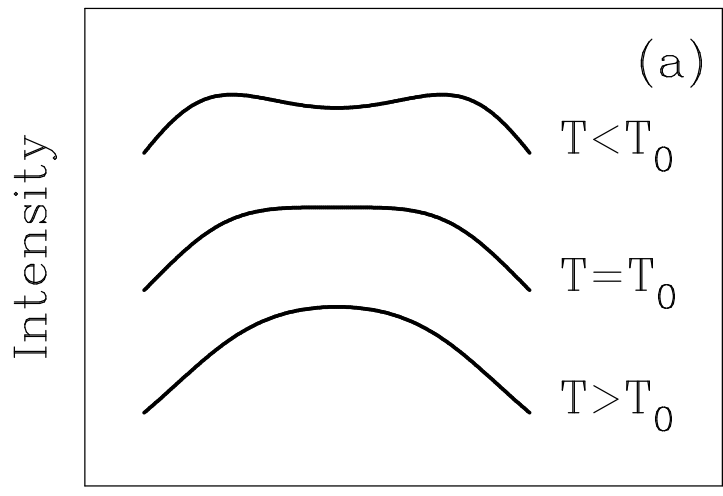

Wavevector

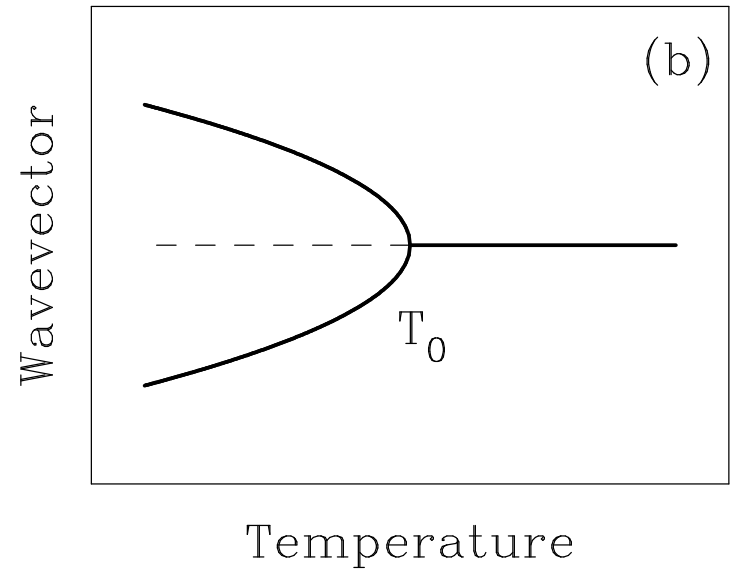

Figure 3. Schematical temperature dependence of the intensity profile (a) and the peak positions (b) in the case of the "thermal" splitting.

This type of behavior was recently experimentally observed (and subsequently reproduced in the MC simulations) for the Pt-V alloy system by Le Bolloc'h et al. ${ }^{37}$ In this system the splitting of the (100) intensity peak along the (h00) line occured with decreasing concentration of vanadium rather then temperature. The explanation of this anomaly was proposed by the author, ${ }^{39}$ who also pointed out that similar splitting should take place when temperature decreases at fixed composition. The predicted effect was then discovered in the MC simulations by the same group. ${ }^{47}$ Experimental confirmation of its existence remains to be seen.

\section{REFERENCES}

1. D. de Fontaine, Solid State Physics 34:73 (1979); 47:33 (1994).

2. F. Ducastelle. Order and Phase Stability in Alloys, North-Holland, Amsterdam (1991).

3. G. Inden and W. Pitsch, in: Phase Transformations in Materials, P. Haasen, ed., VCH Press, New York, (1991).

4. A.G. Khachaturyan. Theory of Structural Transformations in Solids, Wiley, New York, (1983).

5. M. A. Krivoglaz. Theory of X-Ray and Thermal Neutron Scattering by Real Crystals, Plenum, New York (1969); Diffuse Scattering of X-Rays and Neutrons by Fluctuations, Springer, Berlin (1996).

6. J. M. Ziman. Models of Disorder, Cambridge University Press, Cambridge (1979).

7. L.D. Landau and E.M. Lifshitz. Statistical Physics, Part I, Pergamon, Oxford (1980).

8. Yu.A. Izyumov and Yu.N. Skryabin. Statistical Mechanics of Magnetically Ordered 
Systems, Consultants Bureau, New York and London (1988).

9. H. Ehrenreich and L.M. Schwartz, Solid State Phys. 31:149 (1976).

10. R.J. Elliott, J.A. Krumhansl, and P.L. Leath, Rev. Mod. Phys. 46:465 (1974).

11. J.S. Faulkner, Prog. Mater. Sci. 27:1 (1982).

12. V.I. Tokar, I.V. Masanskii, and T.A. Grishchenko, J. Phys. Condens. Matter 2:10199 (1990).

13. I.V. Masanskii, V.I. Tokar, and T.A. Grishchenko, Phys. Rev. B 44:4647 (1991).

14. D.J. Amit. Field Theory, the Renormalization Group and Critical Phenomena, World Scientific, Singapore (1984).

15. A.N. Vassiliev. Functional Methods in Quantum Field Theory and Statistics, Leningrad State University Press, Leningrad (1976).

16. A.N. Vassiliev and R.A. Radzhabov, Teor. Mat. Fiz. 21:49 (1974); 23:366 (1975).

17. N.M. Bogoliubov et al., Teor. Mat. Fiz. 26:341 (1975).

18. P.C. Clapp and S.C. Moss, Phys. Rev. 142:418 (1966); 171:754 (1968).

19. R. Brout. Phase Transitions, Benjamin, New York (1965).

20. J. M. Sanchez, Physica A 111:200 (1982).

21. T. Mohri, J.M. Sanchez and D. de Fontaine, Acta Metall. 33:1463 (1985).

22. G.S. Joyce, in: Phase Transitions and Critical Phenomena, Vol. 2, C. Domb and M.S. Green, eds., Academic Press, New York (1972).

23. J. Philhours and G.L. Hall, Phys. Rev. 170:496 (1968).

24. D.W. Hoffmann, Metall. Trans. 3:3231 (1972).

25. L. Onsager, J. Am. Chem. Soc. 58:1468 (1936).

26. J.B. Staunton and B.L. Gyorffy, Phys. Rev. Lett. 69:371 (1992).

27. V.I. Tokar, Phys. Lett. A 110:453 (1985).

28. V. Janiš, Czech. J. Phys. B 36:1107 (1986); Phys. Rev. B 40:11331 (1989).

29. R. Kikuchi, Phys. Rev. $81: 988$ (1951).

30. I. Tsatskis (unpublished).

31. I. Tsatskis (submitted).

32. V.I. Tokar and I.V. Masanskii, Fiz. Metal. Metalloved. 64:1207 (1987).

33. I.V. Masanskii and V.I. Tokar, Teor. Mat. Fiz. 76:118 (1988).

34. T.A. Grishchenko, I.V. Masanskii, and V.I. Tokar, J. Phys. Condens. Matter 2:4769 (1990).

35. I.V. Masanskii and V.I. Tokar, J. Phys. I France 2:1559 (1992).

36. L. Reinhard and S.C. Moss, Ultramicroscopy 52:223 (1993).

37. D. Le Bolloc'h et al., in: Proceedings of the Joint NSF/CNRS Workshop on Alloy Theory, Mont Sainte Odile Monastery, Strasbourg, France, October 11-15, 1996, Comput. Mater. Sci. 8:24 (1997).

38. M. Borici-Kuqo and R. Monnier, Ref. 37, p. 16.

39. I. Tsatskis (submitted).

40. H. Reichert, S.C. Moss and K.S. Liang, Phys. Rev. Lett. 77:4382 (1996).

41. S.C. Moss and H. Reichert (private communication); H. Reichert, I. Tsatskis and S.C. Moss, Ref. 37, p. 46.

42. H. Roelofs et al., Scripta Mat. 34:1393 (1996).

43. S.C. Moss, Phys. Rev. Lett. 22:1108 (1969); S.C. Moss and R.H. Walker, J. Appl. Crystallogr. 8:96 (1974).

44. I. Tsatskis (in preparation).

45. J. Kulik, D. Gratias, and de D. Fontaine, Phys. Rev. B, 40:8607 (1989).

46. A. Finel and D. de Fontaine, J. Statist. Phys. 43:645 (1986).

47. D. Le Bolloc'h et al. (private communication and in preparation). 